\title{
Long-Tailed Trapping Times and Lévy Flights in a Self-Organized Critical Granular System
}

\author{
Marián Boguñá* and Álvaro Corral ${ }^{\dagger}$ \\ Departament de Física Fonamental, Universitat de Barcelona, Diagonal 647, E-08028 Barcelona, Spain
}

(Received 21 January 1997)

\begin{abstract}
We present a continuous time random walk model for the scale-invariant transport found in a selforganized critical rice pile [K. Christensen et al., Phys. Rev. Lett. 77, 107 (1996)]. From our analytical results it is shown that the dynamics of the experiment can be explained in terms of Lévy flights for the grains and a long-tailed distribution of trapping times. Scaling relations for the exponents of these distributions are obtained. The predicted microscopic behavior is confirmed by means of a cellular automaton model. [S0031-9007(97)03489-3]
\end{abstract}

PACS numbers: 64.60.Lx, 05.40. $+\mathrm{j}, 46.10 .+\mathrm{z}, 64.60 . \mathrm{Ht}$

Self-organized criticality (SOC), or the spontaneous emergence of scale invariance in nonequilibrium systems, has attracted a great interest as an explanation of fractal behavior in nature [1]. Sandpiles rapidly became the paradigm of SOC, but it has been only very recently that they have been shown to be characterized by power-law distributions of avalanches [2,3]. On the other hand, understanding the complex behavior of granular media is a challenge of fundamental physics per se. Between many other amazing properties, granular systems can behave simultaneously as solids or liquids and show a glassy dynamics with extremely slow relaxations [4]. In addition, the transport properties found in granular systems displaying SOC [5] turn out to be very similar to the dispersive transport taking place in amorphous semiconductors and polymers [6]. Finally, there are close connections between sandpile models and interface depinning [7].

The experimental system that we want to analyze is a rice pile, built in the narrow gap between two vertical plates, over a quasi-one-dimensional support of length $L$. Rice grains are added to the left side, where a vertical bar between the two plates forms a wall that keeps the grains inside the system. In contrast, the right side is open, and allows the exit of the grains out of the pile. Starting with an empty system, the slow addition of grains makes the pile grow until the profile reaches the open boundary at the right. After this transient time, the pile arrives at a quasistationary state where the average slope fluctuates around a well defined angle of repose, and the influx of grains at the left equals on average the outflux at the right exit. At this point it has been shown by the Oslo group [2] that the rice pile displays SOC if the shape of the grains is anisotropic enough to prevent the rolling of the grains down the slope, suppressing the inertial effects and enhancing the dissipation by means of the friction. This result, apart from being the first unambiguous evidence of SOC in granular media $[2,3]$, points out the fact that SOC is associated with strongly dissipative systems.

Christensen et al. [5] have studied the transport properties of individual grains through the Oslo rice pile. The transit time of tracer grains, defined as the time necessary for a tracer to escape from the pile, was measured experimentally. The results led to a power-law distribution of transit times for long times preceded by a flat region, i.e.,

$$
P_{\mathrm{tr}}(T) \sim\left\{\begin{array}{l}
\text { constant for small } T, \\
1 / T^{\alpha} \text { for large } T,
\end{array}\right.
$$

with $\alpha=2.4 \pm 0.2$ [5]. Moreover, when the system size is varied, the distribution verifies a finite size scaling,

$$
P_{\text {tr }}(T, L)=L^{-\nu^{\prime}} F\left(T / L^{\nu}\right),
$$

with $\nu=1.5 \pm 0.2$ and $\nu^{\prime}=1.4 \pm 0.2$ [5]. The fact of having $\nu \simeq \nu^{\prime}$ follows from the normalization condition. From here, the scaling of the mean transit time with system size was found to be "very anomalous,"

$$
\langle T\rangle \sim L^{\nu} .
$$

The goal of this Letter is to study the microscopic properties of the transport of grains inside the rice pile, by means of a continuous time random walk model [8]. Comparing our theory with the experimental findings, we can give a form for the distribution of trapping times and the distribution of flights in the real system. In addition, we test our conclusions using a one-dimensional cellular automaton modeling the experiment [5] that connects transport with avalanche dynamics.

We are going to consider the motion of a single grain or particle through the profile as essentially one dimensional. This can be done because in the experiments the path of the particles takes place between two points, it starts at the top of the pile, next to the left wall, and ends at the rightmost extreme of the support. The rice grain remains at rest, trapped at position $x$ during a random time interval $t$ until some avalanche reaches it. At this point the grain performs an instantaneous jump, or flight, of random length $l$, after which it becomes trapped at $x+l$. Then the dynamics of a particle is described in terms of a continuous time random walk, fully specified by the distribution of trapping times $\psi(t)$ and by the distribution of flights $\phi(l)$. To be precise $\psi(t) d t$ is the probability that the particle is trapped at a given position a time between $t$ and $t+d t$, whereas $\phi(l) d l$ gives the probability that 
the particle jumps a distance between $l$ and $l+d l$ during an avalanche. Notice that we can assume $l>0$ always, since in the experiment no mechanism allows the grains to climb the profile, always decreasing to the right. This will be a great simplification in the calculations in comparison with models for diffusion in amorphous semiconductors [9]. In addition, the length of the flight $l$ will not be limited by the system size. Both variables $t$ and $l$ are taken as independent random processes. The assumption of statistical independence has succeeded in reproducing experimental results, for instance, in Ref. [10]. Finally note that with the hypothesis of instantaneous jumps we are nothing else than fulfilling the usual condition for SOC, that is to have a slowly driven system with two separated time scales, where the motion of grains (or avalanches) happens at infinite velocity in comparison with any external time scale.

The magnitude of interest is the distribution of transit times $P_{\text {tr }}(T, \mathcal{L})$, where $P_{\text {tr }}(T, \mathcal{L}) d T$ gives the probability that the particle takes a time between $T$ and $T+d T$ to travel from the origin to position $x=\mathcal{L} \leq L$. In the context of stochastic processes the transit time is, in fact, the first passage time to level $\mathcal{L}$. This distribution can be easily related to $p(x, t)$, defined in such a way that $p(x, t) d x$ is the probability that at time $t$ a particle is in a position between $x$ and $x+d x$, the time being measured since the addition of the particle. The probability of being at $x \leq \mathcal{L}$ at time $t$ is equal to the probability of having a transit time $T>t$; mathematically,

$$
\int_{0}^{\mathcal{L}} p(x, t) d x=1-\int_{0}^{t} P_{\mathrm{tr}}(T, \mathcal{L}) d T .
$$

On the other hand, $p(x, t)$ depends on the renewal density $h(x, t)$, where $h(x, t) d x$ gives the probability of jump per unit time in a position between $x$ and $x+d x$ [11]. We can write close equations relating $p(x, t)$ and $h(x, t)$, using $\psi(t)$ and $\phi(l)$,

$$
\begin{aligned}
h(x, t)= & {[\mu \phi(x)+(1-\mu) \delta(x)] \psi(t) } \\
& +\int_{0}^{t} \int_{0}^{x} h\left(x^{\prime}, \tau\right) \phi\left(x-x^{\prime}\right) \psi(t-\tau) d x^{\prime} d \tau,
\end{aligned}
$$

$$
\begin{aligned}
p(x, t)= & {[\mu \phi(x)+(1-\mu) \delta(x)] \Psi(t) } \\
& +\int_{0}^{t} \int_{0}^{x} h\left(x^{\prime}, \tau\right) \phi\left(x-x^{\prime}\right) \Psi(t-\tau) d \tau d x^{\prime},
\end{aligned}
$$

where $\Psi(t) \equiv \int_{t}^{\infty} \psi\left(t^{\prime}\right) d t^{\prime}$ gives the probability that the particle survives a time larger than $t$ trapped at any position. $\mu$ is the probability that at $t=0$ the particle is moving. Equations (4)-(6) contain the solution to our problem, relating a measurable magnitude, $P_{\mathrm{tr}}(T, \mathcal{L})$, with the magnitudes that define the microscopic dynamics, $\psi(t)$ and $\phi(l)$. Applying the Laplace transform, defined as $\hat{f}(\omega, s) \equiv \int_{0}^{\infty} d x \int_{0}^{\infty} d t e^{-\omega x} e^{-s t} f(x, t)$, the equations become linear and straightforwardly solvable. In particular, $P_{\text {tr }}$ turns out to be

$\hat{P}_{\mathrm{tr}}(s, \omega)=\frac{1}{\omega}\left[1-\frac{[1-\hat{\psi}(s)]\{1-\mu[1-\hat{\phi}(\omega)]\}}{1-\hat{\psi}(s) \hat{\phi}(\omega)}\right]$.

The Laplace transform of the mean transit time $\langle T(\mathcal{L})\rangle$ can be easily obtained from $\hat{P}_{\mathrm{tr}}(s, \omega)$ as

$$
\langle T(\omega)\rangle=-\left[\frac{\partial}{\partial s} \hat{P}_{\mathrm{tr}}(s, \omega)\right]_{s=0}=\frac{1-\mu(1-\hat{\phi})}{\omega(1-\hat{\phi})}\langle t\rangle,
$$

where the existence of $\langle T(\omega)\rangle$ depends directly on the existence of the first moment of $\psi(t),\langle t\rangle \equiv \int_{0}^{\infty} t \psi(t) d t=$ $-(d \hat{\psi} / d s)_{s=0}$. As in the experiment $\langle T(\mathcal{L})\rangle$ was found to be finite, we conclude that $\langle t\rangle$ exists as well. The same reasoning for $\left\langle T^{2}(\mathcal{L})\right\rangle$ that was infinite in the experiment implies that $\left\langle t^{2}\right\rangle$ is not defined, and then we assume

$\psi(t) \sim B / t^{2+\beta}, \quad$ when $t \rightarrow \infty, \quad$ with $0<\beta \leq 1$.

This means that in Laplace space [12]

$$
\hat{\psi}(s) \sim 1-\langle t\rangle_{s}+B \Gamma(-1-\beta) s^{1+\beta} \quad \text { when } s \rightarrow 0,
$$

where $\Gamma(\cdot)$ is the gamma function. Substituting this expression into the equation for $\hat{P}_{\text {tr }}$ (7) and inverting the Laplace transform for $s$ we obtain

$$
\hat{P}_{\mathrm{tr}}(t, \omega) \sim \frac{1-\mu(1-\hat{\phi})}{\omega(1-\hat{\phi})} \frac{B}{t^{2+\beta}},
$$

for $t \rightarrow \infty$. Comparing (9) and (11) we see how the distribution of transit times, that is, a macroscopic quantity, is closely related to the distribution of trapping times, a microscopic magnitude. In fact, both are long-tailed distributions with the same exponent. Linking with the experimental result (1) we can conclude that the trapping times are power law distributed with an exponent

$$
2+\beta=\alpha .
$$

Notice that the tail of the transit time distribution only depends on the trapping time distribution, and not on the distribution of jumps. Alternatively, going back to Eq. (7) we can perform first the long distance limit, i.e., $\omega \rightarrow 0$. If we consider that the jump distribution has mean value but not second moment, that is,

$$
\phi(l) \sim C / l^{2+\gamma} \quad \text { when } l \rightarrow \infty, \quad \text { with } 0<\gamma \leq 1,
$$

then $\hat{\phi}(\omega)$ verifies an equation similar to (10) that substituting on (7) and inverting the Laplace transform gives

$$
\hat{P}_{\text {tr }}(s, \mathcal{L}) \sim \frac{C}{1+\gamma}\left(\mu+\frac{\hat{\psi}}{1-\hat{\psi}}\right) \frac{1}{\mathcal{L}^{1+\gamma}},
$$

when $\mathcal{L} \rightarrow \infty$. This behavior will correspond to times "smaller" than $\mathcal{L}$. For small times, the movement of the 
grains will be shallow and the probability of a given transit time up to a position $\mathcal{L}$ will be independent on system size; in other words, Eq. (14) will not depend on $L$. If we make $\mathcal{L}=L$, this means that the scaling with system size is the same as with position. Denoting the latter by subindices $\nu_{x}$ and $\nu_{x}^{\prime}$, i.e., $P_{\text {tr }}(T, \mathcal{L})=\mathcal{L}^{-\nu_{x}^{\prime}} \mathcal{F}\left(T / \mathcal{L}^{\nu_{x}}\right)$, this implies that $\nu^{\prime}=\nu_{x}^{\prime}$, and comparing with the scaling found in the experiment (2) we have

$$
1+\gamma=\nu^{\prime} \text {. }
$$

One can consider other asymptotic forms for $\phi(l)$, but it is only the one given by Eq. (13) that reproduces an exponent $\nu^{\prime}$ between 1 and 2. From here we can deduce that in the rice pile the scaling of $P_{\text {tr }}$ given by (2) means that the distribution of jumps has a finite mean $\langle l\rangle$ but an infinite variance. This kind of distributions corresponds to Lévy flights [13], and gives rise to a superdiffusive behavior, as one can verify by finding $\hat{p}(\omega, s)$ and from here obtain [similarly as in Eq. (8)] that $\langle x(t)\rangle \sim t\langle l\rangle /\langle t\rangle$ and $\left\langle x^{2}(t)\right\rangle \propto\left\langle l^{2}\right\rangle=\infty$.

Moreover, if the limit $s \rightarrow 0$ is performed in Eq. (14), one obtains the behavior for large times, but smaller than $\mathcal{L}$, that turns out to be independent on $t$,

$$
P_{\mathrm{tr}}(t, \mathcal{L}) \sim \frac{C}{(1+\gamma)\langle t\rangle} \frac{1}{\mathcal{L}^{1+\gamma}}, \quad \text { when } t<\frac{\langle t\rangle}{\langle l\rangle} \mathcal{L} .
$$

This corresponds to the flat region observed in the transit time distribution before the power-law decay. In fact, the appearance of a plateau in $P_{\mathrm{tr}}$ is an indication of the existence of $\langle t\rangle$.

Now that the asymptotic form of $\phi(l)$ is known, one can go back to Eqs. (11) and (8) to perform the long distance limit in order to obtain the scaling of $P_{\text {tr }}$ with $\mathcal{L}$ for large times and the scaling of the mean transit time,

$$
\begin{aligned}
P_{\text {tr }}(t, \mathcal{L}) \sim \frac{B}{\langle l\rangle} \frac{\mathcal{L}}{t^{2+\beta}}, \quad \text { when } t \gg \frac{\langle t\rangle}{\langle l\rangle} \mathcal{L}, \\
\langle T(\mathcal{L})\rangle \sim \mathcal{L}\langle t\rangle /\langle l\rangle, \quad \text { when } \mathcal{L} \rightarrow \infty .
\end{aligned}
$$

From here one obtains that $\nu_{x}=1$, in contrast with the value of $\nu_{x}^{\prime}=1+\gamma$. The reason to have $\nu_{x} \neq \nu_{x}^{\prime}$ is simple: the model does not show finite size scaling for all $T$ and $\mathcal{L}$. Indeed we have found scaling only for a region $T<\mathcal{L}\langle t\rangle /\langle l\rangle$ and $T \gg \mathcal{L}\langle t\rangle /\langle l\rangle$. By using numerical simulations we will see that this behavior is right and one can expect in the experiment $\langle T(\mathcal{L})\rangle \sim \mathcal{L}$ as well. If we impose the continuity of $P_{\mathrm{tr}}$ at the crossover point $T_{c} \sim$ $\mathcal{L}$ we obtain

$$
\beta=\gamma,
$$

that is, $\psi(t)$ and $\phi(l)$ must have the same power-law tail. Employing also Eqs. (12) and (15) we get

$$
\alpha=1+\nu^{\prime} \text {. }
$$

This equation relates the exponent of the power-law tail of $P_{\text {tr }}$ with its scaling with system size, and it is well fulfilled by the experimental values of Ref. [5].
It would be difficult to design an experiment to measure the distributions of trapping times and jumps that are microscopic magnitudes, although in granular materials microscopic and macroscopic scales are not so well separated as in the usual states of matter [4]. As an alternative to support our predictions we use the cellular automaton model introduced in Ref. [5], which was found to reproduce the transport properties of grains quite well. We believe that similar results can be obtained for similar models [14]. On a one-dimensional lattice, from $x=1$ to $L$, an integer variable $h_{x}$ gives the height of the pile at position $x$. Defining the local slope at $x$ as $z_{x} \equiv h_{x}-h_{x+1}$ the dynamics of the model is fully determined by the following rules: if $z_{x} \leq z_{x}^{c} \quad \forall x \Rightarrow z_{1} \rightarrow z_{1}+1$ (a grain is added); if $z_{x}>z_{x}^{c}$ and $x<L \Rightarrow z_{x-1} \rightarrow z_{x-1}+1$, $z_{x} \rightarrow z_{x}-2, z_{x+1} \rightarrow z_{x+1}+1$, and $z_{x}^{c} \rightarrow \operatorname{rand}(1,2)$; if $z_{L}>z_{L}^{c} \Rightarrow z_{L-1} \rightarrow z_{L-1}+1, z_{L} \rightarrow z_{L}-1$, and $z_{L}^{c} \rightarrow$ rand $(1,2)$; where all the sites have to be updated in parallel and rand(1,2) means 1 or 2 at random, with equal probability. The external input of grains at $x=1$ sets the time unit.

The results of Ref. [5] show that Eqs. (1)-(3) are still valid, but the exponents are determined with more accuracy. We reanalyze these results to obtain $\alpha=2.21 \pm$ $0.05, \nu=1.25 \pm 0.10$, and $\nu^{\prime}=1.25 \pm 0.10$, in concordance with (20). The distribution of trapping times can be obtained from simulations as the number of trappings of a given duration divided by the total number of trappings. The results are displayed in Fig. 1. Indeed we obtain a power-law distribution for long times, where the exponent turns out to be $2+\beta=2.20 \pm 0.05$, in very good agreement with our prediction (12) if we compare with the independent measure of $\alpha$. One can also measure the distribution of flight lengths. The behavior for long distances corresponds indeed to a power law, whose exponent is $2+\gamma=2.13 \pm 0.05$, see Fig. 2, where we

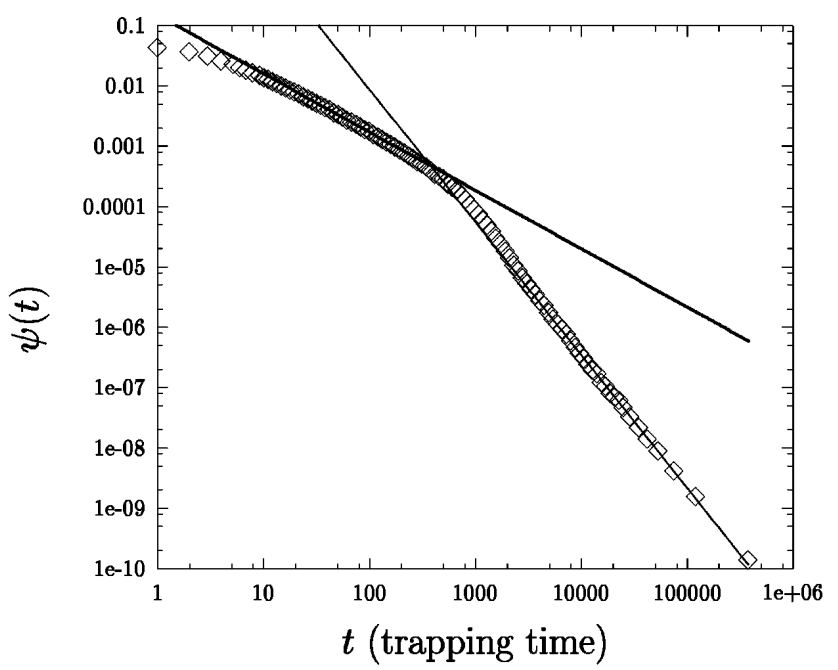

FIG. 1. Trapping time distribution $\psi(t)$ in a system of size $L=400$. The two straight lines are power laws with exponents $-0.97 \pm 0.05$ and $-2.20 \pm 0.05$. 


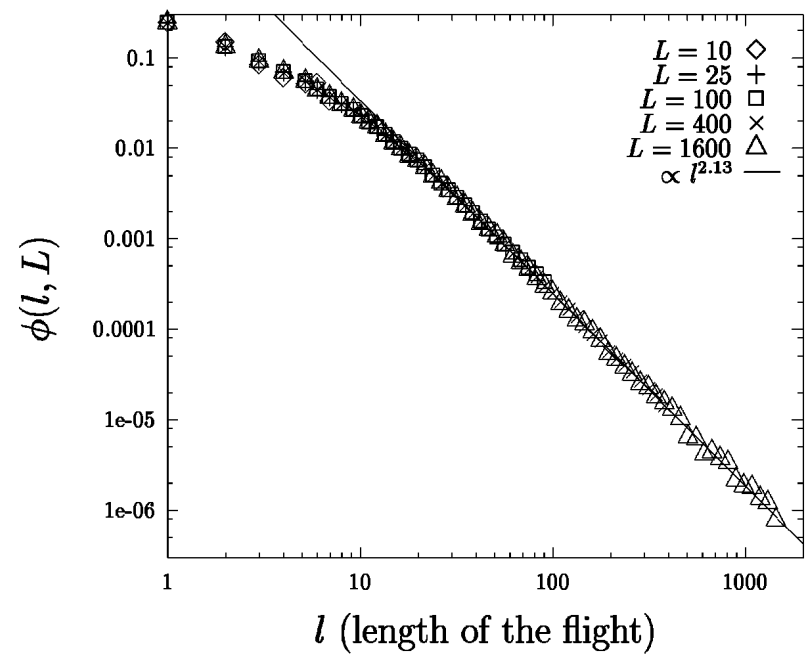

FIG. 2. Distribution of flight lengths $\phi(l, L)$ for different system sizes starting from position $x=2$. The length of the flight does not depend on $L$. The power-law behavior for large distances is characterized by an exponent $-2.13 \pm 0.05$.

restrict the measure to flights starting at a fixed position. Observe from here that $\nu^{\prime}, \beta$, and $\gamma$ are compatible with (15) and (19).

The simulations allow one also to study the transit time to reach a certain position $x=\mathcal{L}$ smaller than the system size $L$. Keeping fixed $L$ the exponents of the scaling of $P_{\text {tr }}$ with $\mathcal{L}$ are $\nu_{x}=1.0 \pm 0.1$ and $\nu_{x}^{\prime}=1.2 \pm 0.1$, as was predicted by Eqs. (16) and (17), whereas the power-law exponent $\alpha=2.18 \pm 0.05$ is in good accord with (20). See Fig. 3. One can additionally measure the mean transit time as a function of $\mathcal{L}$ to verify that Eq. (18) is fulfilled.

Moreover we can derive an additional scaling relation for the model. In Ref. [5] it was found that $\chi=\nu-1$,

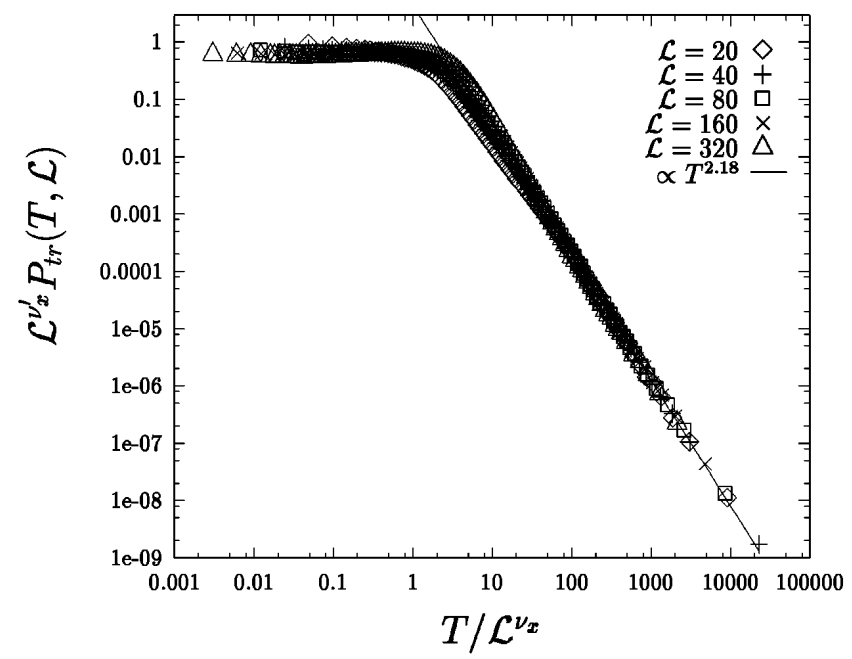

FIG. 3. Scaled distributions of transit times from $x=1$ to $x=\mathcal{L}$ in a system of size $L=400$ for different values of $\mathcal{L}$. The scaling is done for $T \gg \mathcal{L}$ and $T<\mathcal{L}$, resulting that the exponents are $\nu_{x}=1.0$ and $\nu_{x}^{\prime}=1.2$, whereas the power-law exponent is $2.18 \pm 0.05$. Notice that the region with $T$ slightly larger than $\mathcal{L}$ does not scale well. being $\chi$ the roughness exponent of the profile of the pile, that is, the fluctuations of the profile scale as $L^{\chi}$. On the other hand, in Ref. [7] it was argued that $D=2+\chi$, with $D$ the fractal dimension of the avalanches, i.e., the size of the avalanches scales as $L^{D}$. As $\nu \simeq \nu^{\prime}$, combining these relations with (20) one gets

$$
\alpha=D \text {. }
$$

Taking $D=2.23 \pm 0.03$ [7] this last result is in a fair agreement with the measured value of $\alpha$.

In summary, from analytical results and computer simulations we present a coherent scenario for the transport in a self-organized critical granular system. The scale invariance of the process is associated with long-tailed trapping time distributions and Lévy flights of the grains.

It is a pleasure to acknowledge K. Christensen and J. M. Porrà for discussions and suggestions. M. B. is partially supported by CICyT's research program No. PB96-0188, whereas A.C. was supported by No. PB94-0897 and a grant of the Spanish MEC.

Note added. - The measurement of $\psi(t)$ can be difficult but not impossible. We have become aware of recent experiments in good agreement with our results [15].

*Electronic address: mbogunya@hermes.ffn.ub.es

†Electronic address: alvaro@ulyses.ffn.ub.es

[1] P. Bak, C. Tang, and K. Wiesenfeld, Phys. Rev. Lett. 59, 381 (1987); Phys. Rev. A 38, 364 (1988).

[2] V. Frette, K. Christensen, A. Malthe-Sørenssen, J. Feder, T. Jøssang, and P. Meakin, Nature (London) 379, 49 (1996); see also M. Kardar, Nature (London) 379, 22 (1996).

[3] J. Feder, Fractals 3, 431 (1995).

[4] H. M. Jaeger, S. R. Nagel, and R. P. Behringer, Phys. Today 49, No. 4, 32 (1996); Rev. Mod. Phys. 68, 1259 (1996).

[5] K. Christensen, A. Corral, V. Frette, J. Feder, and T. Jøssang, Phys. Rev. Lett. 77, 107 (1996).

[6] H. Scher, M.F. Schlesinger, and J.T. Bendler, Phys. Today 44, No. 1, 26 (1991).

[7] M. Paczuski and S. Boettcher, Phys. Rev. Lett. 77, 111 (1996).

[8] E. W. Montroll and G. H. Weiss, J. Math. Phys. (N.Y.) 6, 167 (1965); G. H. Weiss, Aspects and Applications of the Random Walk (North-Holland, Amsterdam, 1994).

[9] M. F. Schlesinger, J. Stat. Phys. 10, 421 (1974); H. Scher and E. W. Montroll, Phys. Rev. B 12, 2455 (1975).

[10] E. Morales-Gamboa, J. Lomnitz-Adler, V. RomeroRochín, R. Chicharro-Serra, and R. Peralta-Fabi, Phys. Rev. E 47, R2229 (1993).

[11] D. R. Cox, Renewal Theory (Methuen, London, 1962).

[12] N. Bleistein and R. A. Handelsman, Asymptotic Expansions of Integrals (Dover, New York, 1986), p. 134.

[13] J. Klafter, M.F. Shlesinger, and G. Zumofen, Phys. Today 49, No. 2, 33 (1996).

[14] L. N. Amaral and K. B. Lauritsen, Phys. Rev. E 54, R4512 (1996); (unpublished).

[15] A. Malthe-Sørenssen (private communication). 